\title{
Minimizing the Detent Force in Permanent Magnet Linear Synchronous Motor for driving of 2D Laser Marking Table
}

\author{
Lyubomir Lazov ${ }^{1}$, Peter Uzunov ${ }^{2}$ \\ Latvia Academy of Technologies, Rezekne, Latvia ${ }^{1}$ \\ Electricity System Operator, Sofia, Bulgaria ${ }^{2}$
}

\begin{abstract}
In this paper the research results for reducing the detent force in one innovative permanent magnet linear synchronous motor for $2 D$ laser marking system was published. There two methods are used. The first of these methods features the usage of two additional end teeth with chamfers in the magnetic circuit of the movable part. In the second method, the teeth of the ferromagnetic core are with different lengths. As a result of the change of the air gap permeance in both cases substantial reduction of detent force is achieved, in multiples at times. The results obtained are based on modeling and analyzing the linear motor magnetic field by the Finite Element Method (FEM). Provided experimental research of the linear motor prototype proves the correctness of the simulations results.
\end{abstract}

Keywords: Laser Marking, Permanent Magnet Linear Synchronous Motors, Detent force, Finite Element Analysis.

\section{INTRODUCTION}

Nowadays, linear motors are meant to replace a system using a rotating motor and a transmission to realize a linear movement. With linear motors the performances increase considerably since the mechanical limitations are removed. This leads to a better precision, a higher acceleration and a higher speed of the moving part. Therefore, direct drives with linear motors are increasingly used in industrial applications although these solutions need often more investment costs.

In [1] the design of PMLSM drive table for laser marking system of small details has been described. This is one flat type iron core Permanent Magnet Linear Synchronous Motor (PMLSM).

Its dynamics and control are hindered by the presence of detent force.

Due to permanent magnets (PM), a high force of attraction between the supply part and the magnetic way appears. For the same propulsion force, the ratio between the attractive force and the propulsion force is about 5 times without additional cooling. Furthermore, due to the teeth in the mover core, this type of motor can have a higher cogging force.

The force of attraction between the iron-core of the primary and the PM of the secondary is known as the cogging force and it occurs in both the rotational and linear machines. In linear machines, there is an additional force due to the finite length of the mover, which is called the end-effect force [2].

The end-effect force is caused only by the interaction of the end teeth with magnets, whereas the cogging force is produced by all teeth along the iron core.

Both the cogging force and the end-effect force combine to produce the net detent force of a PMLSM. Mathematically,

$$
F_{\text {det ent }}=F_{\text {cogging }}+F_{\text {endeffect }} \text {. }
$$

Detent force generates pulsations in propulsion force and causes disturbances in the control of PMLSM. Therefore, measures must be taken for its reduction.

For this reason, special techniques for minimizing this detent force are used. One of them is the usage of additional teeth end on both sides of the ferromagnetic core [3]. Another way for minimizing the cogging force is the usage of teeth of ferromagnetic core with unequal length [4].

In this paper the both techniques for the minimizing the detent force are analyzed. The analysis of the detent force in both cases is very difficult to perform with an analytical method and therefore FEM is used to show their impact on the detent force. Detent force was calculated on the base of the results from FEA of the motor magnetic field. As this magnetic field in both cases is flat parallel, 2D FEM was used for the simulations.

The 3D CAD model of PMLSM under study in this paper is shown in Fig.1 [1].

The main technical specifications of the linear servomotor are given in Table I.

In this article, the research results are published for decreasing the detent force of this PMLSM which

ISSN 1691-5402

(C) Rezekne Academy of Technologies, Rezekne 2017 http://dx.doi.org/ 10.17770/etr2017vol3.2651 
drives the two-coordinate table to which details for laser marking are affixed.

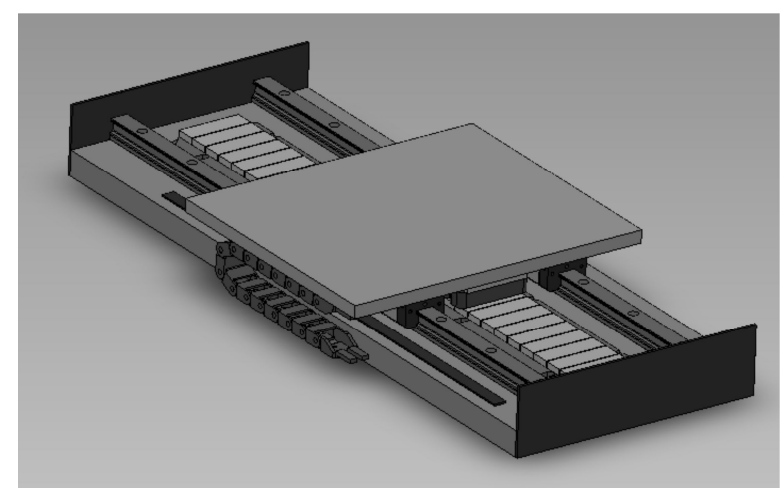

Fig. 1. 3D CAD model of PMLSM

\section{II.FEM MODELING}

The motor magnetic field modeling has for basics the Maxwell's laws. Here the static magnetic field exited only from PMs in 2D case is modeled.

The general differential form of the Maxwell's equations is:

$$
\begin{gathered}
\vec{\nabla} \times \vec{H}=\vec{J} ; \\
\vec{B}=\mu \vec{H} ; \\
\vec{\nabla} \vec{B}=0 ;
\end{gathered}
$$

where: $\vec{H}$ is the vector of magnetic field; $\vec{B}$ is the vector of magnetic flux density.

Table I

Specifications of the test bench

\begin{tabular}{|l|l|l|}
\hline $\mathrm{N}$ & Quantity & Value \\
\hline 1 & Number of phases & 3 \\
\hline 2 & Number of poles & 10 \\
\hline 3 & Slot number & 12 \\
\hline 4 & Rated phase voltage (V) & 50 \\
\hline 5 & $\begin{array}{l}\text { Magnetic flux density in teeth and } \\
\text { core yoke (T) }\end{array}$ & 1.6 \\
\hline 6 & Sizes of permanent magnets (mm) & $76.2 / 12.7 / 6.35$ \\
\hline 7 & Air gap (mm) & 1 \\
\hline 8 & Pole pitch (mm) & 15 \\
\hline 9 & Tooth pitch (mm) & 12.5 \\
\hline 10 & Rated phase current (A) & 9.0 \\
\hline 11 & Propulsion force (N) & 180 \\
\hline 12 & Speed (m/s) & 3 \\
\hline 13 & Mover mass (kg) & 2.0 \\
\hline 14 & Efficiency & 0.85 \\
\hline 15 & Power factor & 0.9 \\
\hline & & \\
\hline
\end{tabular}

Furthermore, from (3) by applying one particular property of vector calculation the magnetic potential vector $\vec{A}$ is defined as:

$$
\vec{B}=\operatorname{rot} \vec{A}
$$

If a material is nonlinear, the permeability $\mu$, is actually a function of $\vec{B}$ :

$$
\mu=\frac{\vec{B}}{\vec{H}(\vec{B})} .
$$

Then, (1) can be rewritten as [5]:

$$
\vec{\nabla} \times\left(\frac{1}{\mu(\vec{B})} \vec{\nabla} \times \vec{A}\right)=\vec{J}
$$

For a linear isotropic material (and assuming the Coulomb gauge, $\vec{\nabla} \vec{A}=0$ ), (6) reduces to:

$$
-\frac{1}{\mu} \vec{\nabla}^{2} \vec{A}=\vec{J}
$$

In the general 3-D case, $\vec{A}$ is a vector with three components. However, in the 2-D planar and axisymmetric cases, two of these three components are zero, leaving just the component in the "out of the page" direction.

The advantage of using the vector potential formulation is that all the conditions to be satisfied have been combined into a single equation. If $\vec{A}$ is found, $\vec{B}$ and $\vec{H}$ can then be deduced by differentiating $\vec{A}$. The form of (6), an elliptic partial differential equation, arises in the study of many different types of engineering phenomena.

The distribution of linear motor static magnetic field can be found by solving the equation (7) with the program FEMM [5].

FEMM retains the form of (6), so that magnetostatic problems with a nonlinear $B-H$ relationship can be solved.

The problem was solved in area, shown on Fig. 2.

The problem was solved under Dirichelet boundary conditions applied to outside border of the model - the circle around the geometry of the linear motor, as was shown in Fig. 2.

$$
\left.\vec{A}\right|_{K}=0
$$

These boundary conditions allow the simulation of the linear motor motion. For this purpose, in the program written in GUI Octave software [6], a loop over the position of the motor mover within the limits of two pole pitches $2 \tau_{\mathrm{p}}$ has been used. The position step $x_{\text {step }}$ used in simulation was calculated as follows 


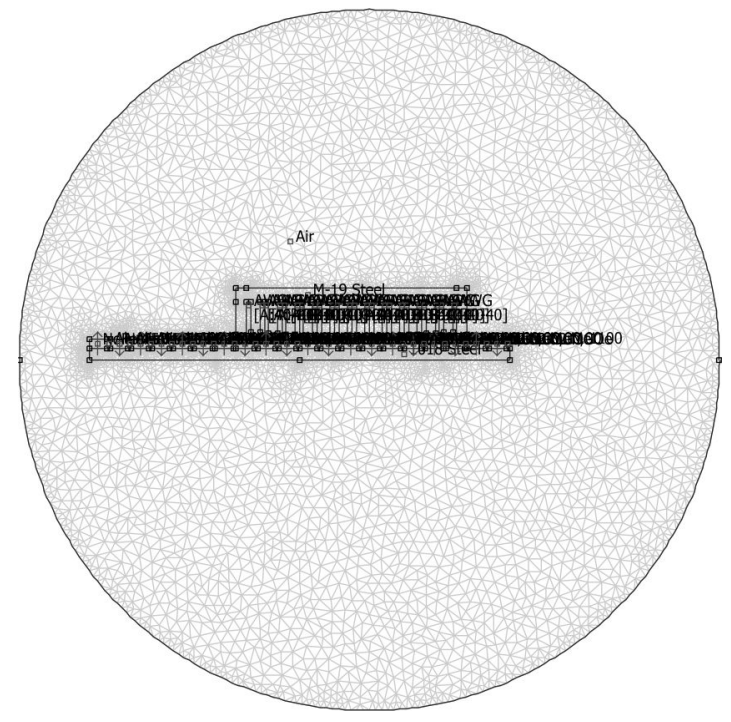

Fig. 2. The solution area for FEA

$$
x_{\text {step }}=\frac{\tau_{p}}{n},
$$

where $n$ is the number of steps.

The problem domain was discretized on the FEM mesh with 30727 nodes and 61092 triangle finite elements (Fig.2).

For each mover position, Finite Element Analysis (FEA) of the motor magnetic field at no-load (Fig. 3) were performed and on the base of results obtained by Maxwell tensor, force components over the motor moving part along both axes $\mathrm{Ox}$ and $\mathrm{Oy}$ were calculated.

The force component along axis Ox really represents the detent force that opposes the motor thrust.

The detent force versus mover position for the PMLSM designed in [1] without any measures for its reduction is shown in graphical form in Fig.6 for $b_{\mathrm{z}}=$ $0 \mathrm{~mm}$.

\section{DETENT FORCE MINIMIZING}

A. Using additional two end teeth with chamfer

This method is described in many references [7]. In accordance with it, in the ferromagnetic core two additional teeth 1 and 2 are formed as shown in Fig.4. Furthermore, these teeth have chamfers 3 and 4.

With this ferromagnetic core geometry new FEM model was made. On the base of the magnetic field analysis results the detent force of the linear motor was calculated. The calculation results show a decreasing of the detent force.

This is due to the fact that the permeance of the magnetic flux changes in this manner at the end regions of the mover core, thus improving the magnetic field distribution (Fig. 5).

To establish the optimal width of the end teeth and the chamfer angle, field analyses are made for models wherein tooth width changes with specific steps for certain chamfer angles. When the optimal width of the teeth at the ends of the core is established, the angle of the chamfer starts varying until its optimal value is set. The criterion is the minimum value of the detent force.

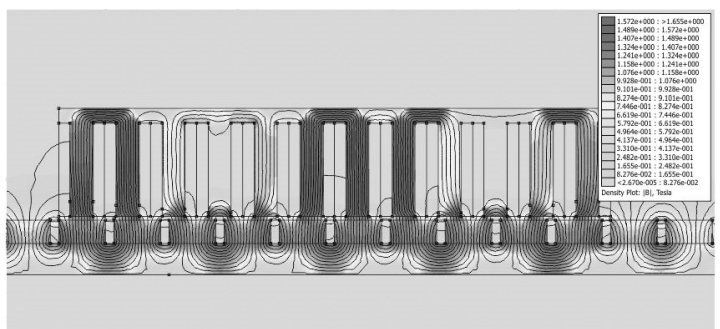

Fig. 3. The motor magnetic field distribution in final loop step

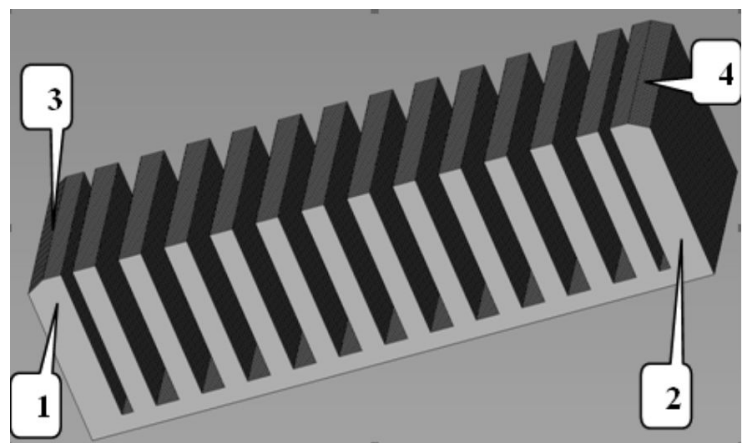

Fig.4. 3D CAD model of mover core

The results from the optimization of the width $b_{z}$ of the additional teeth are shown in graphical form in Fig.6.

Calculations shows that for a mover core having thickness of additional teeth $b_{\mathrm{z}}=10 \mathrm{~mm}$, a good reduction of the detent force is obtained. But then, attraction force increases from $3431 \mathrm{~N}$ per $3968 \mathrm{~N}$. This effect leads to unnecessary load on the linear guide of the mover and shortens the service life of the linear motor as a whole.

This method of reducing the detent force is characterized by the fact that the additional teeth lead to greater weight of the movable part. Furthermore, increase of strength of attraction between the permanent magnets and the ferromagnetic core of the movable part is obtained.

\section{B. Using different length of the teeth of mover} core

In [4] an alternative method to reduce the detent force was presented. For this purpose, ferromagnetic core of the movable part is used wherein the teeth are with different length - progressively decreasing in the direction of travel and symmetrically in the opposite direction, as shown in Fig. 7.

The determination of the step which reduces the length of the teeth is the task of FEA of the motor magnetic field.

For this purpose, a new FEM model was made, shown in Fig. 8. 


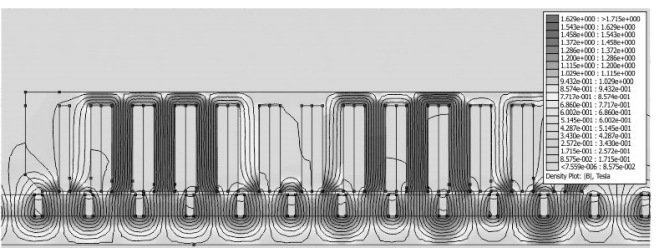

Fig. 5. Magnetic flux density distribution in the case of mover core with two additional end teeth

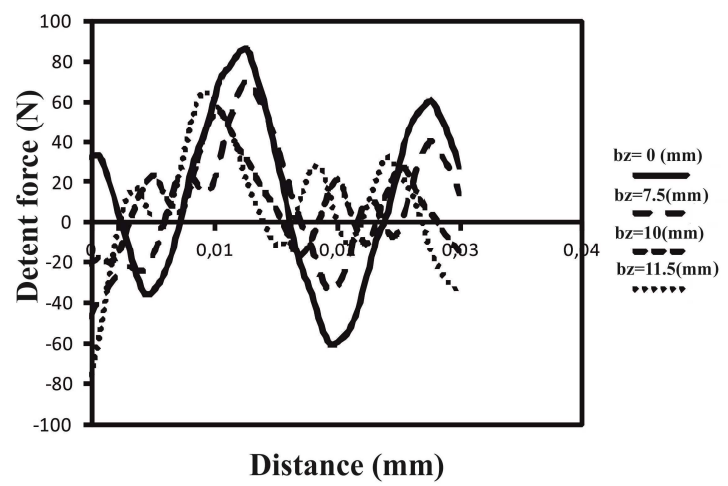

Fig.6. Detent force in relation to the additional teeth width

The analysis of the motor magnetic field at different mover positions to the PMs was performed and the detent force in each step was calculated. Fig. 9 shows the field distribution for the mover displacement $x=30 \mathrm{~mm}$.

It is important to clarify the question for the size of step for reducing the teeth length. The results from the detent force calculations for two-step reduction of the teeth length - 0.5 and $1 \mathrm{~mm}$ - are shown in Fig. 10 .

The research shows that in the case of reducing the final four teeth of mover core near its two ends with step size $1 \mathrm{~mm}$, significant reduction of the detent force is achieved.

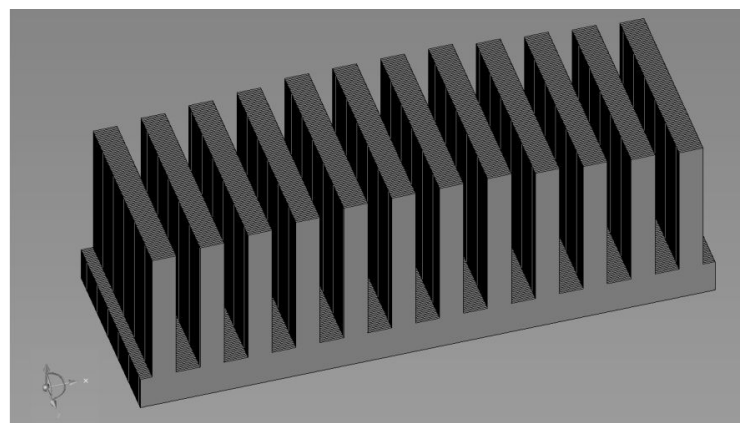

Fig.7. Mover ferromagnetic core with teeth with progressively decrease

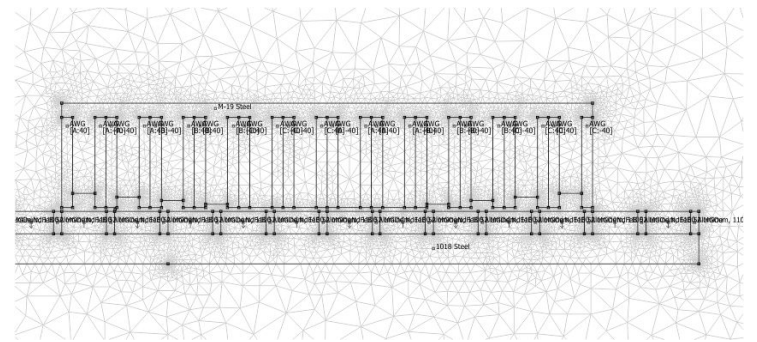

Fig.8. FEM model of the linear motor with a mover ferromagnetic core with teeth of different lengths

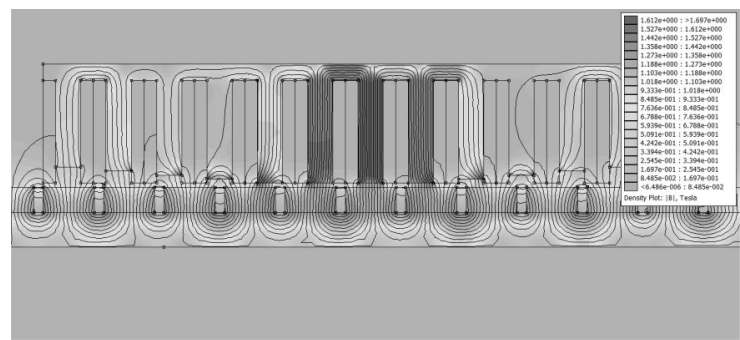

Fig.9. Distribution of the magnetic field density at the mover position $x=30 \mathrm{~mm}$

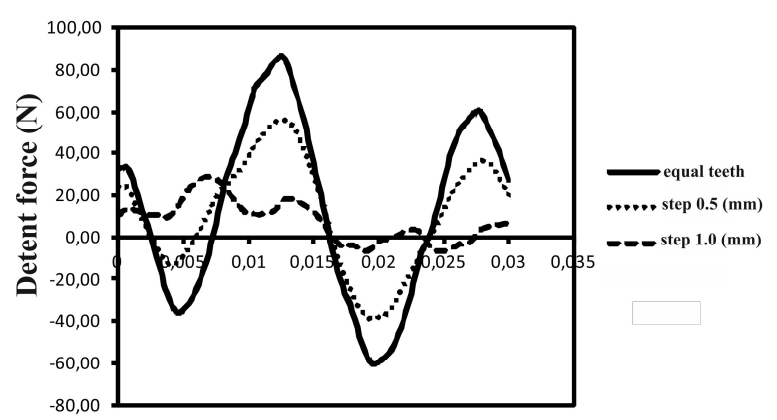

Distance (mm)

Fig.10. Detent force relation to step size 0.5 and $1 \mathrm{~mm}$, for decreasing the teeth length

\section{CONCLUSIONS}

From FEM simulations of the PMLSM drive laser marking table in this paper it was found out that the reduction of the detent force can be achieved by using two additional teeth at both ends of the mover ferromagnetic core formed with a chamfer and by using teeth of the mover core with different lengths. The greater weight of the movable part in the case with two additional teeth and the bigger force of attraction between PMs and the mover core are respectively a reason for worsening the dynamic performance of the linear motor. For these reasons, this method for detent force reduction will be not used in final prototype of researched linear synchronous servomotor. Hence, a second way ferromagnetic mover core with decreasing length of its teeth - was used. Thus the permeance of motor air gap was changed and in result pulsations and amplitude of the detent force was reduced. The absence of additional final two teeth and reducing the weight of ferromagnetic core in this case improved the motor dynamics.

\section{REFERENCES}

[1] P. Uzunov, L. Lazov, "Design of Permanent Magnet Linear Synchronous Motor driving 2D Table for Laser Marking", Presented of 11th International Scientific and Practical Conference. Environment. Technology. Resoures. Rezekne Academy of Technologies, Rezekne, Latvia, 2017.

[2] Chevailler S., "Comparative study and selection criteria of linear motors", Ph.D. Thesis, EPFL, Suisse, 2006. 
Environment. Technology. Resources, Rezekne, Latvia Proceedings of the $11^{\text {th }}$ International Scientific and Practical Conference. Volume III, 174-178

[3] Remy, G., G.Krebs, A. Tounzi, P.Barre, "Finite Element Analysis of a PMLSM (part 1) - Meshing techniques and thrust computations", LDIA 2007, Lille, France, Sept. 2007.

[4] A. Jenny, "Iron core linear motor having low detent force with high power density", US Patent 7,687,942 B2, March 30, 2010.

[5] D. Meeker, "FEMM reference manual", 2015. [Online]. Available: http://www.femm.info/ Archives/doc/
[6] J. Varandas,"GUI Octave",2011. [Online]. Available: http://download.cnet.com/windows/joaquim-varandas/326020_4-10128082-1.html

[7] Gomand G.,Remy J., A. Tounzi, Pierre-Jean Barre, "Impact of currents on the detent force of a PMSLM", Proceedings of EPNC 2008, Lille, France, July 2-4, 2008. 